\title{
Efficient wireless sensor deployment at minimum cost
}

\author{
Francesca Guerriero ${ }^{1}$, Luigi Di Puglia Pugliese ${ }^{1}$, and Nathalie Mitton ${ }^{2}$ \\ 1 Department of Mechanical, Energy and Management Engineering, University of Calabria, Italy \\ \{francesca.guerriero.luigi.dipugliapugliese\}@unical.it \\ 2 Inria \\ nathalie.mitton@inria.fr
}

\begin{abstract}
We address the problem of defining a wireless sensor network by deploying sensors with the aim of guaranteeing the coverage of the area and the connectivity among the sensors. The wireless sensor networks are widely studied since they provide several services, e.g., environmental monitoring and target tracking. We consider several typologies of sensors characterized by different sensing and connectivity ranges. A cost is associated with each typology of sensors. In particular, the higher the sensing and connectivity ranges, the higher the cost. We formulate the problem of deploying sensors at minimum cost such that each sensor is connected to a base station with either a one- or a multi-hop and the area is full covered. We present preliminary computational results by solving the proposed mathematical model, on several instances. We provide a simulation-based analysis of the performances of such a deployment from the routing perspective.
\end{abstract}

Keywords: sensor deployment · routing · wireless · cost efficiency

\section{Introduction}

These last years have witnessed the development of several wireless sensors deployment strategies, to be applied in many different fields. A large part of them focus on environmental monitoring and surveillance operations [4]. When deploying such a sensor network, the following main challenges arise. How many sensors need to be deployed to ensure the full area coverage at a minimum cost? Where to deploy them to keep not only the proper monitoring of the area, but also to ensure the existence of a wireless multi-hop path between any pair of nodes in the network? Then, once sensors deployed, how good is the quality of the communicating network yet constructed beyond the plain connectivity?

Covering problems have been investigated for several years by different research communities: robotics, networking and optimization groups. Most of robotics researchers mainly couple coverage with path planning and friction efforts; networking researchers mainly consider energy costs and connectivity while optimization researchers have generally neglected the connectivity issues. To the best of our knowledge, only very few approaches $[11,12,2]$ from the literature consider jointly connectivity and quality of monitoring coverage. These approaches propose mixed integer linear programs to formulate the problem of deploying sensors ensuring coverage and connectivity. Efficient solutions are obtained, achieving very interesting and performing results, but they do not take into account the deployment cost. In addition, the solutions obtained by using the existing approaches are considered satisfactory if they achieve connectivity, whereas none of them evaluates the quality of the communication network obtained.

In this paper, we assume to have a limited number of sensors of different typologies, each characterized by a sensing range, a communication range and an operational cost. We add communication constraints in order to force sensors to be connected (permanently or intermittently) and form a communication network, allowing them to dynamically self-adapt to any environment aspect. We also consider the energy consumption and the travelled distance.

The main contributions of our work are reported in what follows:

- We address a generalized sensors deployment problem with coverage and connectivity constraints, considering different typologies of sensors.

- We formulate the problem as a mixed integer program and we provide a deep evaluation of the solutions obtained, analyzing the efficiency and the effectiveness with respect to the deployment cost.

- We assess the obtained sensor deployment, in terms of quality of communication topology. 
The remainder of this paper is organized as follows. Section 2 summarizes the state of the art. In Section 3 we formally describe the addressed problem along with the mathematical formulation. Section 4 provides the numerical results collected on meaningful instances. Section 5 concludes the paper.

\section{State of the art}

The coverage and monitoring of a point or an area of interest have been studied by different research communities. i.e., networking, robotics, and optimization.

The robotics community refers to the techniques set up to cover an area as "formation control". They generally do not consider connectivity issues $[14,16]$.

In the optimization field, some works focus on multi-objective mathematical models, to determine the best placement of mobile nodes for different tasks; we cite, for example, the papers of Guerriero et al. [5] and Lambrou et al. [8]. Guerriero et al. [5] focus on the optimal sensor displacement to allow the network to achieve high performance in terms of energy consumption and travelled distance.

In Lambrou et al. [8], the authors provide a collaborative architecture between mobile and static sensors. The coverage objective described in [8] is the same as the one taken into account in the present work. However, they do not consider the communication constraints. Yu et al. [17] applied Genetic Particule Swarm Optimization (GA-PSO) techniques to solve the optimal sensor deployment problem. However, the authors do not pursue the same goal as in this paper and do not address communication aspects.

Several challenging issues arise when quality of coverage and connectivity issues are considered simultaneously [7]. From the networking perspective, self-deployment distributed and localized protocols are generally proposed to address these specific aspects. These protocols broadly aim at a single objective which is either cover a point of interest/target [6] or a whole area of interest [1], that might be mobile [10]. Such approaches used different tools such as Virtual Forces [13], Swarm Optimization [9] or graph theory tools $[3,15]$.

Very few works, addressing jointly communication connectivity and quality of coverage, have been published by researchers operating in the optimization field. The main goal considered in $[11,12,2]$ is very similar to the one taken into account in the present paper, since the authors aim at covering a sensing area, by deploying the minimum number of wireless sensors, while maintaining the connectivity between the deployed sensors. In $[11,12]$, the authors address the problem by reducing it to a two-dimensional critical coverage problem (an NP-Complete problem), and developing an integer linear programming formulation to obtain an optimal solution. In [2] standard mixed integer linear programs are used to represent several variants of the problem. Although these works achieve very interesting results, they do not consider the cost of the deployment.

Most of the existing approaches that consider the cost of a wireless sensor network only focus on the deployment phase (cost of a sensor, number of sensors and sometimes distance to drop it), but, to the best of our knowledge, none of them take into account the operational cost (energy sensors will consume to send and forward data) and quality (quality of links).

\section{Problem definition}

Given a field, the objective is to deploy a set of sensors at minimum cost, such that full coverage and full connectivity are ensured.

We assume the availability of $K$ different typologies of sensors. Each sensor $k \in K$ is characterized by a sensing range $r_{k}$, a communication range $R_{k}$ and a cost $c_{k}$. In addition, we assume a limited number of sensors for each typology, i.e., $n_{k}$. We assume a point is covered if it is within a sensing range of at least one sensor.

The full connectivity is guaranteed if each sensor is connected to a sink node $o$ with either a 1-hop or multi-hop communication. In the latter case, the other sensors play the role of relay.

We formulate the problem over a complete graph $G(N, A, C)$ where $N$ is the set of possible location points in the field where the sensors could be deployed, $A$ is the set of arcs connecting each point $i \in N$ to the other points $j \in N \backslash\{i\}, C$ is the set of locations to be covered (so that are in sensing range of at least one sensor). The Euclidean distance $d_{u v}$ is associated with each pair of positions $u, v \in N \cup C$. 


\subsection{Mathematical formulation}

To formulate the problem, we introduce the following decision variables:

$x_{i j}, \forall(i, j) \in A$. Continuous variables taking value greater than zero if a communication between position $i$ and $j$ is active, zero otherwise.

$w_{i j}, \forall(i, j) \in A$. Binary variables taking value equal to one if a bilateral communication between $i$ and $j$ can be activated, i.e., $d_{i j} \leq \min \left\{R_{k}, R_{k^{\prime}}\right\}$, where $k$ and $k^{\prime}$ are the typologies of sensors located in position $i$ and $j$, respectively, zero otherwise.

$y_{i}^{k}, \forall i \in N, k \in K$. Binary variables that assume value equal to one if a sensor of typology $k$ is deployed in position $i$, zero otherwise.

$z_{h}^{i}, \forall h \in C, i \in N$. Binary variables taking value equal to one if point $h$ is covered by a sensor deployed in position $i$, zero otherwise.

The problem can be formulated as follows:

$$
\begin{aligned}
& \min \sum_{i \in N} \sum_{k \in K} c_{k} y_{i}^{k} \\
& \text { s.t. } \\
& \sum_{i \in N} y_{i}^{k} \leq n_{k}, \quad \forall k \in K, \\
& \sum_{k \in K} y_{i}^{k} \leq 1, \quad \forall i \in N, \\
& w_{i j} \leq \frac{\sum_{k \in K} y_{i}^{k} R_{k}}{d_{i j}}, \forall(i, j) \in A, \\
& w_{i j} \leq \frac{\sum_{k \in K} y_{j}^{k} R_{k}}{d_{i j}}, \forall(i, j) \in A, \\
& x_{i j} \leq M w_{i j}, \quad \forall(i, j) \in A, \\
& \sum_{(i, j) \in A} x_{i j}-\sum_{(j, i) \in A} x_{j i}=\left\{\begin{array}{l}
\sum_{k \in K} \sum_{j \in N} y_{j}^{k} \quad \text { if } i=o \\
-\sum_{k \in K} y_{i}^{k}
\end{array} \quad\right. \text { otherwise } \\
& z_{h}^{i} \leq \frac{\sum_{k \in K} y_{i}^{k} r_{k}}{d_{i h}}, \forall h \in C, i \in N, \\
& \sum_{i \in N} z_{h}^{i} \geq 1, \forall h \in C, \\
& x_{i j} \in \mathbb{R}_{+}^{|A|}, w_{i j} \in\{0,1\}, \forall(i, j) \in A, y_{i}^{k} \in\{0,1\}, \forall i \in N, k \in K, \\
& z_{h}^{i} \in\{0,1\}, \forall i \in N, h \in C .
\end{aligned}
$$

Equation (1) minimizes the cost of deployed sensors. Equation (2) allows the deployment of at most $n_{k}$ sensors for each typology $k \in K$. Equation (3) imposes that at most one sensor is deployed for each position $i \in N$. Equations (4) and (5) define the variables $w_{i j}$. In particular, $w_{i j}$ is imposed to be zero in the case either the distance between the sensors deployed in positions $i$ and $j$ is greater than the minimum communication range between both sensors or no sensor is deployed in either position $i$ or $j$. Equations (6) ensure that each arc $(i, j)$ is active if and only if it is able to connect sensors deployed in positions $i$ and $j$, where $M$ is a big positive number. If $w_{i j}=1$ means that the sensors deployed at positions $i$ and $j$ can communicate. Since the value of $x_{i j}$ is not upper bounded, then it can assume any positive value $\leq M$. Equations (7) represent flow constraints aiming at defining the communication between the sink node $o$ and all the deployed sensors. Equations (8) define the coverage of position $h \in C$. In particular, if the sensing range of a sensor of typology $k$ located in position $i$ is less than the distance $d_{h i}$ between $h \in C$ and $i \in N$, then variable $z_{h}^{i}$ is forced to assume the value zero. Equations (9) impose that each point $h \in C$ is covered by at least one sensor. Equation (10) defines the domain of the decision variables. We observe that equations (7) represent flow conservation constraints. Once a solution is determined, variables $x_{i j}$ define a possible communication tree among the deployed sensors. It is as if we considered that each sensor sent one unit of data, thus all variables $x_{i j}>0$ define the quantity of data that traverses 
$\operatorname{arc}(i, j)$. In the worst case, the data of all deployed sensors are sent through a single arc. It follows that the value that each variable $x_{i j}$ can assume is upper bounded by $\min \left\{|N|, \sum_{k \in K} n_{k}\right\}$. The value of $M$ can be set to this value,i.e., $M=\min \left\{|N|, \sum_{k \in K} n_{k}\right\}$.

We highlight that the proposed model can couple the case where a target has to be covered by at least $S>1$ sensors. Indeed, it is sufficient to introduce the parameter $S$ in the right-hand-side of constraint (9) for the point that needs to be covered by the $S$ sensors (possibly all points).

\section{Computational results}

In what follows, we analyze the behaviour of the proposed mathematical model, referred in the sequel as GMc, on randomly generated instances.

We have considered also the case in which the number of sensors is minimized. In particular, the objective function (1) has been modified in $\sum_{i \in N} \sum_{k \in K} y_{i}^{k}$. The model with the latter objective function is referred in the sequel as GMs.

The models have been implemented in Java language and solved by CPLEX 12.51. The tests were carried out on an Intel(R) core(TM) i7-4720HQ CPU 2.60GHz 8GB RAM machine.

The main aim of this section is to present the numerical results obtained and to show the bi-objective nature of the considered problem, with the respect to the minimization of the overall cost, i.e., function (1) and the minimization of the number of deployed sensors (i.e., $\sum_{i \in N} \sum_{k \in K} y_{i}^{k}$ ). We suppose that the higher the sensing range, the higher the cost. On the one hand, when minimizing the number of sensors, those with higher sensing range are chosen, on the other hand, sensors with low sensing range are preferred because they have lower cost than those with high sensing range. The numerical results confirmed the conflicting nature of the two objectives. A sensitivity analysis on the number of available typologies of sensors is also carried out. Finally, we evaluate the effectiveness of the solutions from a communication point of view.

We deploy a varying number of nodes at position $<x, y>$ such that $X_{\min } \leq x \leq X_{\max }$ and $Y_{\min } \leq y \leq Y_{\max }$ in a field with varying size.

The results are collected on instances generated by considering the following characteristics:

$-X_{\min }=Y_{\min }=0$

$-X_{\max }=Y_{\max } \in\{10,12,14,20\}$

$-n_{k}=100, \forall k \in K$

- each point $i \in N$ has integer coordinates $\left(X_{i}, Y_{i}\right)$ in the defined field

The testing phase has been carried out by considering four scenarios that differ from each other for the dimension of the (square) field, i.e., $(10,10),(12,12),(14,14)$, and $(20,20)$. The instances are characterized by the following parameters:

$-|K| \in\{2,3,4,5\}$

$-r_{k}$ randomly chosen in the interval $[2,6]$

- $R_{k}=R * r_{k}$ with $R=\max \{1, \alpha f\}, \alpha \in[0,1], f=\sqrt{3}$ meaning that $1 \leq \frac{R_{k}}{r_{k}} \leq \sqrt{3}$

$-c_{k}=r_{k}^{2}$

- each point $h \in C$ has coordinates associated to the centre of the cell. Each corner is a point in $N$.

- the sink is located at coordinate $(0,0)$

We follow the scientific literature to generate the instances. The dimensions of the field and the sensing range values refer to [2], whereas, the connectivity range values satisfy the property described in [7]. Indeed, $\frac{R_{k}}{r_{k}} \leq \sqrt{3}$ guarantees that the full coverage does not imply the full connectivity. Fixing the number of typologies of sensors, we generate 5 different configurations varying the seed for the randomly generated parameters.

\subsection{Numerical results}

In this section, we analyze the results obtained by solving GMc, aimed at minimizing the overall cost, and the model GMs in which the objective function, to be minimized, represents the number of deployed sensors (i.e., $\sum_{i \in N} \sum_{k \in K} y_{i}^{k}$ ). 
First of all, we analyze the efficiency of the proposed model and give some insights on the conflicting nature of the two criteria, i.e., cost and number of sensors. Secondly, we analyze the solutions obtained by both GMc and GMs under a communication point of view. In particular, we investigate how good the network topology built by the sensor deployment is for routing protocols, in terms of path diversities and energy consumption.

Models evaluation. The results obtained with MGs and MGc are shown in Table 1. We report, for each dimension of the field and number of typologies of sensors $|K|$, the number of deployed sensors under column \#sensors, the cost, i.e., the value of (1), under column cost, the execution time of CPLEX for solving the instances in seconds under column time. We impose a time limit of 1800 seconds to the solver for solving each instance, for both models. The superscript near to the time indicates the number of instances that are not solved to optimality within the imposed time limit. We notice that each line of Table 1 reports average results over the five instances generated by varying the seed.

Row AVG reports the average results on the instances with the same dimension of the field, row AVG2 shows the average results over the instances with the same value of $|K|$, and row AVG3 reports the average results over all instances.

\begin{tabular}{|c|c|c|c|c|c|c|c|}
\hline & & & GMc & & & GMs & \\
\hline & $|K|$ & nsors & cost & time & ensors & $\operatorname{cost}$ & time \\
\hline & 2 & 8.80 & 54.00 & $953.36^{2}$ & 3.20 & 75.40 & 1.28 \\
\hline & 3 & 6.40 & 53.00 & $749.78^{2}$ & 2.60 & 71.00 & 1.08 \\
\hline & 4 & 10.20 & 54.00 & $1800.00^{5}$ & 2.20 & 79.20 & 1.42 \\
\hline & 5 & 8.20 & 43.80 & $1494.98^{4}$ & 2.20 & 79.20 & 1.80 \\
\hline AVG & & 8.40 & 51.201 & $1249.53^{13}$ & 2.55 & 76.20 & 1.39 \\
\hline & 2 & 9.80 & 76.20 & $1433.52^{3}$ & 4.60 & 110.80 & 23.82 \\
\hline & 3 & 8.20 & 77.40 & $1528.67^{4}$ & 3.60 & 100.80 & 57.80 \\
\hline & 4 & 11.80 & 75.40 & $1800.00^{5}$ & 3.00 & 99.60 & 71.88 \\
\hline & 5 & 10.80 & 68.60 & $1800.00^{5}$ & 3.20 & 104.20 & 126.51 \\
\hline AVG & & 10.15 & 74.401 & $1640.55^{17}$ & 3.60 & 103.85 & 70.00 \\
\hline & 2 & $1 \%$ & 110.20 & $1800.00^{5}$ & 5.20 & 124.40 & $387.56^{1}$ \\
\hline & 3 & 11.60 & 104.00 & $1800.00^{5}$ & 4.60 & 130.60 & 112.57 \\
\hline & 4 & 12.00 & 117.40 & $1800.00^{5}$ & 4.40 & 158.40 & 173.58 \\
\hline & 5 & 13.80 & 101.00 & $1800.00^{5}$ & 4.20 & 151.20 & 135.83 \\
\hline AVG & & 12.90 & 108.151 & $1800.00^{20}$ & 4.60 & 141.15 & $202.38^{1}$ \\
\hline & 2 & 44 & 284.20 & $1800.00^{5}$ & 0 & 554.20 & $1684.00^{4}$ \\
\hline & 3 & 36.40 & 388.80 & $1800.00^{5}$ & 11.40 & 319.60 & $1800.00^{5}$ \\
\hline$(20,20)$ & 4 & 22.60 & 262.60 & $1800.00^{5}$ & 10.20 & 367.20 & $1800.00^{5}$ \\
\hline & 5 & $32.00 \mathrm{~s}$ & 239.00 & $1800.00^{5}$ & 9.80 & 352.80 & $1800.00^{5}$ \\
\hline AVG & & 28.852 & 293.651 & $1800.00^{20}$ & 13.25 & 398.45 & $1771.00^{19}$ \\
\hline & 2 & 14.30 & 131.151 & $1496.72^{15}$ & & 20 & $524.16^{5}$ \\
\hline & 3 & 15.65 & 155.801 & $1469.61^{16}$ & 5.55 & 155.50 & $492.86^{5}$ \\
\hline & 4 & 14.15 & 127.351 & 1800.0 & 4.95 & 176.10 & $511.72^{5}$ \\
\hline & 5 & 16.20 & 113.101 & $1723.75^{19}$ & 4.85 & 171.85 & $516.04^{5}$ \\
\hline AVG3 & & 15.08 & & $1622.52^{70}$ & 6.00 & 179.91 & $511.20^{20}$ \\
\hline
\end{tabular}

Table 1: Average results obtained with GMc and GMs, varying the dimension of the field and the number of available typologies of sensors.

The results reported in Table 1 highlight that the model GMc is more difficult to solve than GMs. Indeed, 13, 17, 20, and 20 instances with the field $(10,10),(12,12),(14,14)$, and $(20,20)$ are not solved to optimality by the solver within the time limit, respectively. We highlight that 20 instances are considered for each field dimension. Thus, more than a half of instances are not solved for each field dimension. For 
the biggest ones, the optimal solution is not available for all the instances. It follows that solving GMc requires 3.17 times higher computational time than that needed by the solver to handle GMs, on average (see row AVG3).

Comparing the performance of the two models varying the dimension of the field, we observe that the higher the field dimension the lower the difference in term of computational effort between GMc and GMs. Indeed, GMs is 897.53, 23.44, 8.89. and 1.02 times faster than GMc for the fields $(10,10)$, $(12,12),(14,14)$, and $(20,20)$, respectively. It is worth noting that these results are highly influenced by the imposed time limit. Indeed, GMs is able to obtain the optimal solution for all the instance, excluding the one with field $(14,14)$ and the 9 with field $(20,20)$. In addition, solving GMs requires, on average, 1.39, 70.00, 202.38, and 1771.00 seconds. Thus, a high increase in the computational effort is observed. This trend is not evident for GMc since the time limit imposes an upper bound on the computational cost. The same conclusion can be drawn from the results obtained varying the values of $|K|$. In this case, GMc is 2,86, 2.98, 3.52, and 3.34 times slower than GMs for $|K|$ equals to 2, 3, 4, and 5, respectively.

Table 1 highlights the conflicting nature of the two objective functions, i.e., the minimization of the cost and the minimization of the number of sensors.

On the one hand, GMs provides solutions with 6.00 deployed sensors and cost equals to 179.91, on average. On the other hand, the solutions obtained by solving GMc are characterized by 15.08 deployed sensors with a cost of 131.85 , on average. Thus, GMc provides solutions that are 1.56 less expensive than those obtained with GMs, but they require 2.51 times more sensors than those deployed by GMs.

This is an expected trend since the higher the coverage $r$, the higher the cost. Thus, when the number of sensors is minimized, those with higher values of $r$ are preferred with a consequent increase in the cost. On the other hand, when the cost is minimized, sensors with low values of $r$ are chosen. This implies the use of a higher number of such sensors, in order to guarantee the coverage of the field.

Referring to the number of available typologies of sensors $|K|$, we do not observe a clear trend of the difference, in terms of cost, between GMc and GMs (see row AVG2 for each value of $|K|$ of Table 1). Indeed, the solutions obtained by solving GMs are, on average, 1.65, 1.00, 1.36, and 1.52 times more expensive that those obtained by solving GMc for $|K|$ equals to 2, 3, 4, and 5, respectively. This behaviour is due to the fact that GMs does not provide the optimal solution for almost all the instances with field $(20,20)$. This implies that the number of sensors deployed is not minimized, then the cost tends to decrease (by the conflicting nature of the two objectives). As a matter of this fact, if we consider only the fields $(10,10),(12,12)$, and $(14,14)$, the difference in terms of cost between GMc and GMs increases when $|K|$ increases. Indeed, GMs provides solutions that are 1.29, 1.29, 1.37, and 1.57, times more expensive than those provided by GMc for $|K|$ equal to 2,3 , 4, and 5, respectively.

Considering the number of deployed sensors, the higher the value of $|K|$, the higher the difference between the two models (see row AVG2 for each value of $|K|$ of Table 1 ). Indeed, GMs provides solutions with a number of deployed sensors that is $1.65,2.82,2.86$, and 3.34 times lower than that deployed by the solutions obtained by GMc, on average, for values of $|K|$ equals to 2, 3, 4, and 5, respectively.

Topology evaluation. The set of sensors yet deployed aims to efficiently cover and monitor the full area and to constitute a connected network. This property ensures that the base station can communicate with every single sensor and vice-versa. However, this communication is multihop and relies on different routing protocols. In this section, we evaluate the properties of the topologies obtained in the previous sections globally and with regards to the communication and routing costs. We have compared the topologies obtained previously using a home-made $\mathrm{C}$ simulator.

Figure 1 shows the average number of nodes selected by each model for several numbers of available typologies of sensors. We can observe that as expected, the number of nodes selected increases with the area size. Naturally, the solutions obtained with GMs present less nodes than observed for those provided by GMc but also (not shown on the figure), it tends to select only one kind of sensors, the ones with the longer ranges, unlike the GMc that selects several types of sensors to cover the full area at a better cost.

Figure 2 shows the average degree of nodes, i.e., the average number of nodes a node can directly communicate with. It tends to increase with the size of the area. This is due to the fact that the area is bounded and to cover all single small area in the border, we need to increase the density over these points. The node degree increases quicker with GMs than with GMc, which makes GMc potentially more scalable. The mean degree obtained with GMs is very low (between 1 and 2 neighbors in average), which also implies a single communication path between two nodes. This can be a cause of unreliability since 


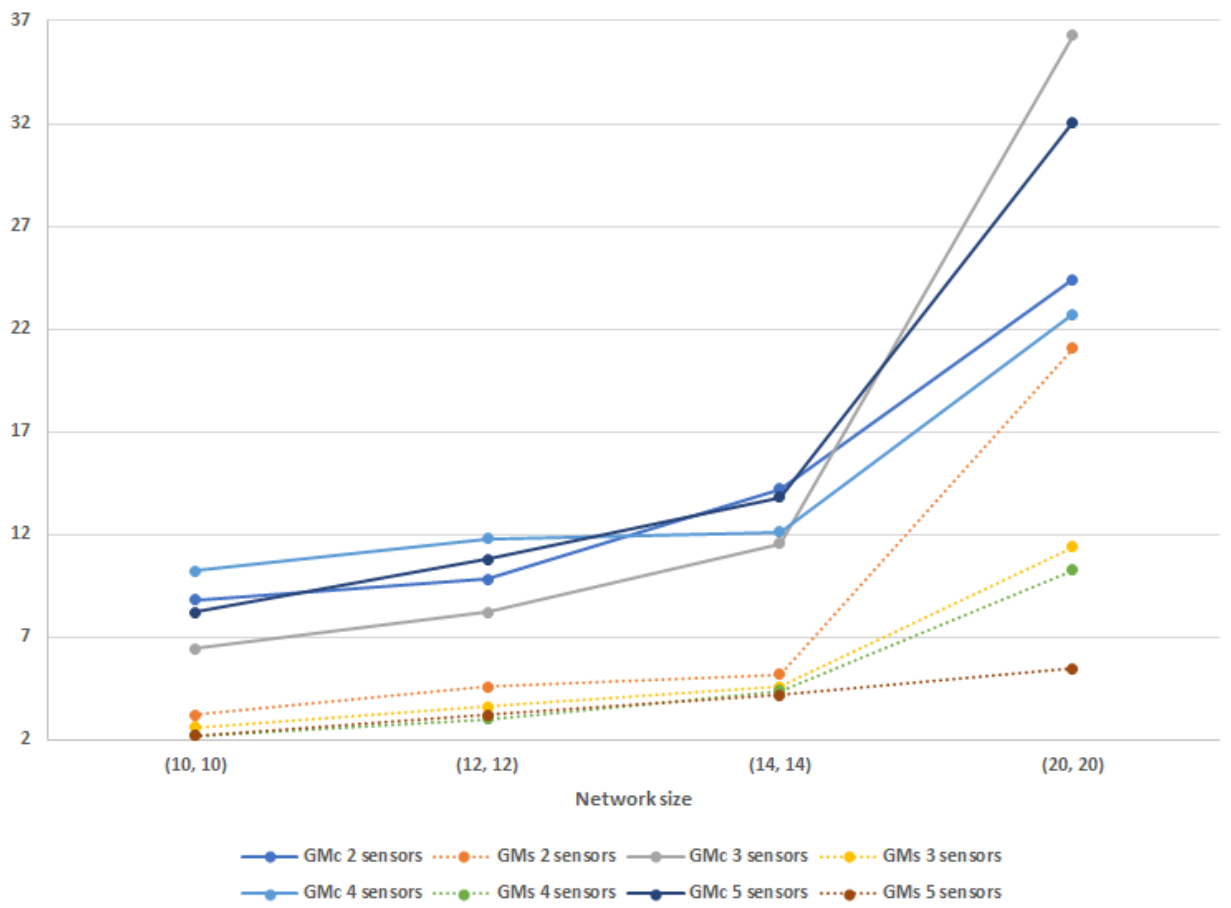

Fig. 1: Average number of nodes varying the field dimension.

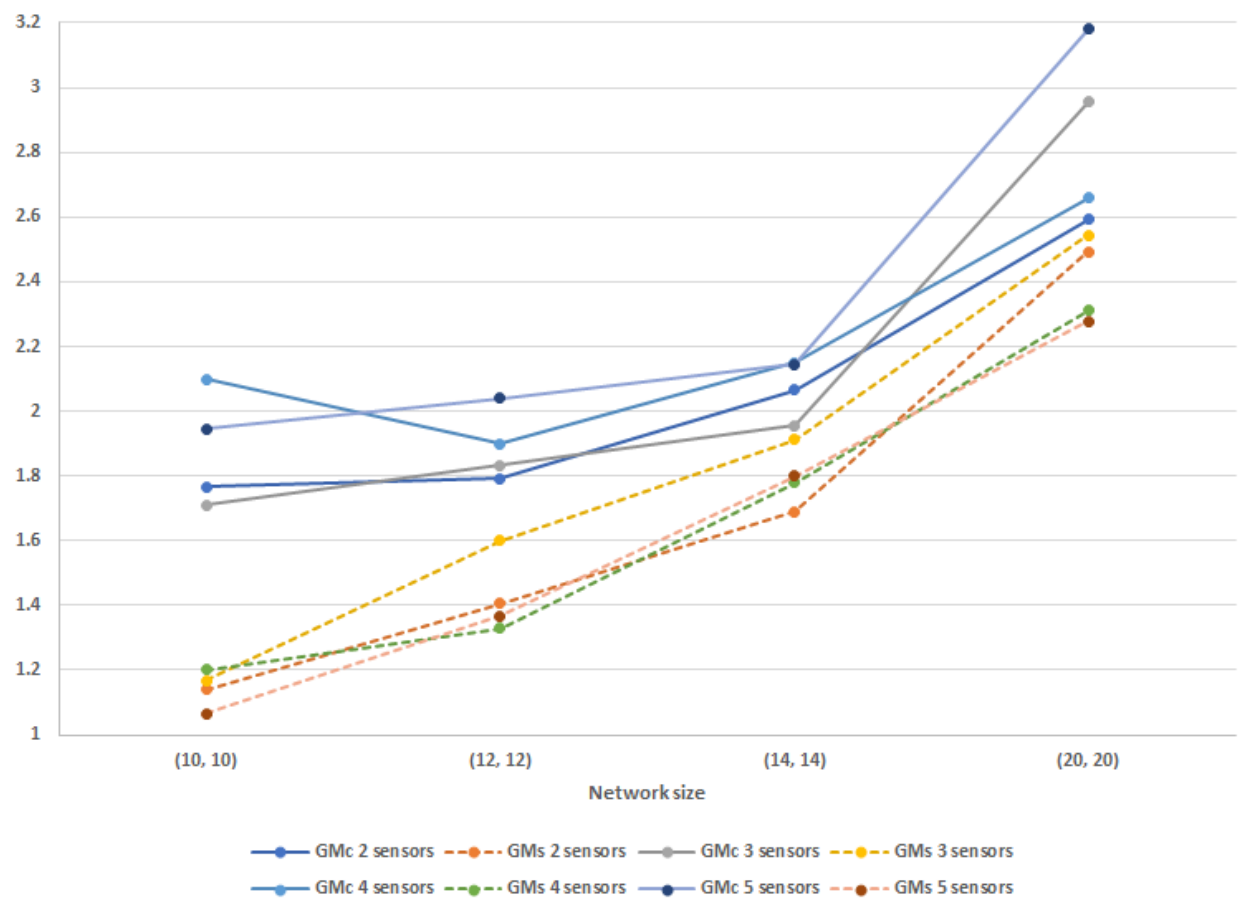

Fig. 2: Average node degree varying the field dimension. 


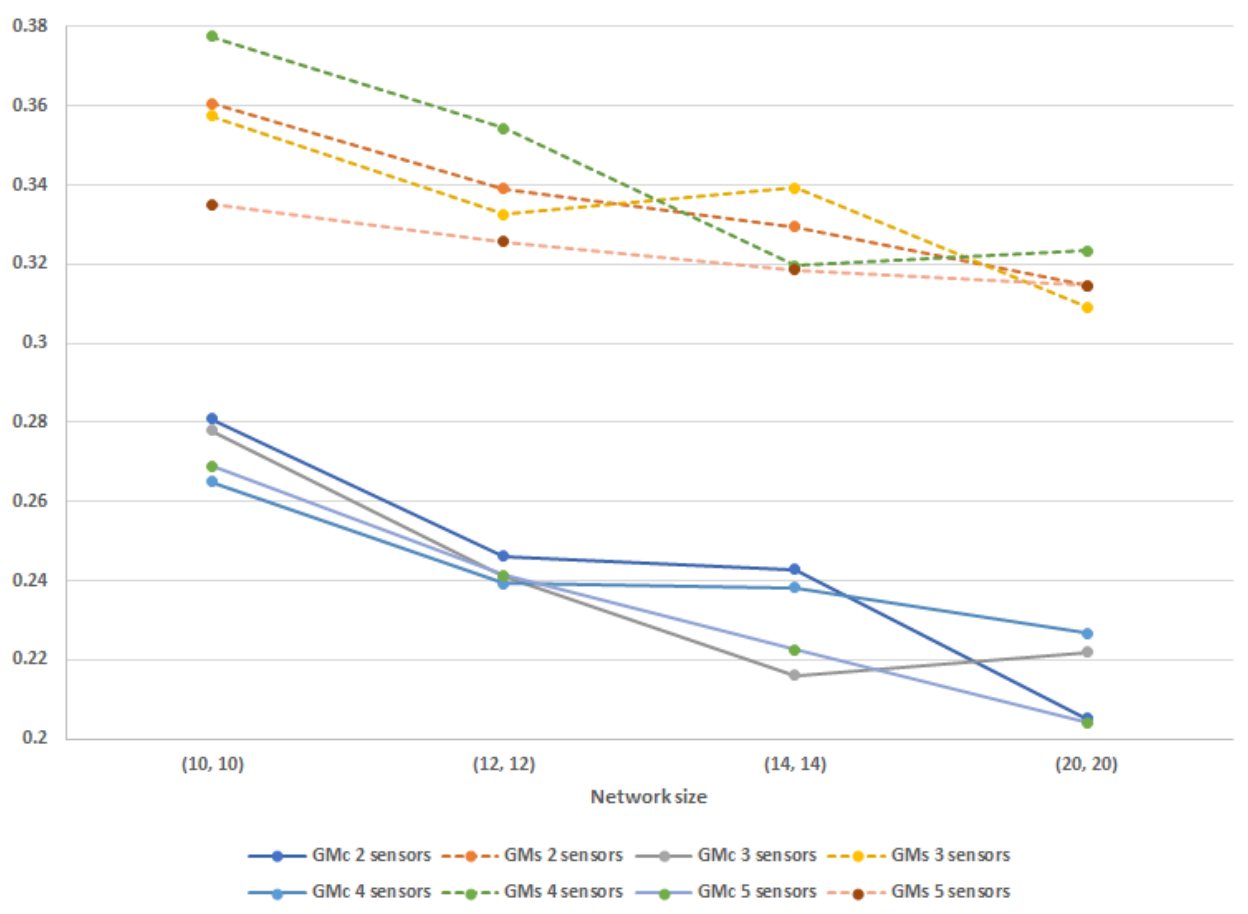

Fig. 3: Average edge length varying the field dimension.

all traffic will take the same path and provokes potential bottlenecks. Each node represents a point of failure on the path since there is no possible alternative path.

Figure 3 shows the average length edge. Obviously, since there are less nodes in the solution provided by GMs than in GMc over a same area, the edges are longer in average with GMs. Thus, they are thus more energy-consuming.

Figures 4 and 5 show some topology examples obtained by model GMc and GMs, respectively, for different number of sensor typologies. In particular, we depict the solutions to the instances with field dimension $(20,20)$ and $(14,14)$ for GMc and GMs, respectively. The edges represent the possible communication among the deployed sensors. Each color corresponds to a typology of sensor.

Although the deployment cost could be reduced with GMs since there are less sensors to deploy, the solutions obtained with the GMc ensure a better cost operation. First, it has been designed to provide the lowest operation cost but also, the topologies that GMc generates provide a better basis for routing data between the different nodes, offering more routing options and lower energy consumption for data transmission.

\section{Conclusions and future work}

In this paper, we have addressed a generalized deployed sensors problem with coverage and connectivity constraints, considering different typologies of sensors. We have formulated the problem as a mixed integer program and we provide a deep evaluation of the solutions obtained, analyzing the efficiency and the effectiveness with respect to the deployment cost. We have compared the solutions obtained with those determined by minimizing the number of deployed sensors. Since the cost increases with the increase of the sensing range, the objectives of minimizing the cost and that of minimizing the number of sensors are in conflict one with the other. Finally, we have assessed the obtained sensor deployment from a quality of communication topology perspective. The numerical results suggest that the solutions obtained when the cost is minimized present a high potential in terms of quality of communication. To the best of our knowledge, we are the first to propose such a study. Our model can be easily extended to ensure a k-coverage of every point (each point of the grid is in sensing range of at least $\mathrm{k}$ different sensors). As a future work, we will investigate further the impact of the so-built topology on routing utilisation together with the global cost (deployment and hardware cost together with software cost). 


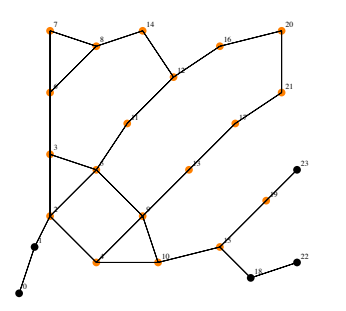

(a) GMc with $|K|=3$

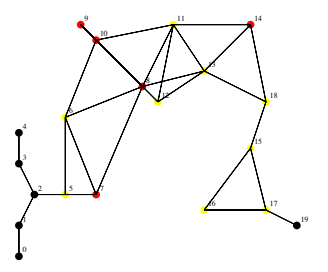

(b) GMc with $|K|=4$

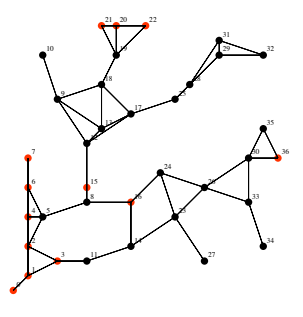

(c) GMc with $|K|=5$

Fig. 4: Topologies obtained with GMc for different numbers of sensor typologies.

\section{References}

1. J. N. Al-Karaki and A. Gawanmeh The Optimal Deployment, Coverage, and Connectivity Problems in Wireless Sensor Networks: Revisited vol 5. 2017.

2. S. Elloumi, O. Hudry, M. Estel, A. Martin, A. Plateau and S. Rovedakis Optimization of wireless sensor networks deployment with coverage and connectivity constraints. Annals of Operations Research, 2018, DOI: $10.1007 / \mathrm{s} 10479-018-2943-7$

3. M. Erdelj, T. Razafindralambo, D. Simplot-Ryl Covering Points of Interest with Mobile Sensors. IEEE Transactions on Parallel and Distributed Systems 24(1), 2013, pp 32-43

4. M. Farsi1, M. A. Elhosseini, M Badawy, H. A. Ali, H. Z. Eldin. Deployment Techniques in Wireless Sensor Networks, Coverage and Connectivity: A Survey. IEEE Access 7, 2019, pp. 28940-28954.

5. F. Guerriero, A. Violi, E. Natalizio, V. Loscri and C. Costanzo. Modelling and Solving Optimal Placement problems in Wireless Sensor Networks. Applied Mathematical Modelling 35 (1), 2011, pp. 230-241.

6. D. Kaur, M. Kaur An Approach of Mobile Wireless Sensor Network for Target Coverage and Network Connectivity with Minimum Movement International Research Journal of Engineering and Technology (IRJET), Vol. 04 (01), 2017

7. I. Khoufi, P. Minet, A. Laouiti, S. Mahfoudh. Survey of Deployment Algorithms in Wireless Sensor Networks: Coverage and Connectivity Issues and Challenges. International Journal of Autonomous and Adaptive Communications Systems (IJAACS), 2017, 10 (4), pp.341-390.

8. T. P. Lambrou and C. G. Panayiotou. Collaborative area monitoring using wireless sensor networks with stationary and mobile nodes. EURASIP Journal on Advances in Signal Processing 2009 (7), pp. 1-16.

9. A. P. Laturkar and P. Malathi Coverage Optimization Techniques in WSN using PSO: A Survey International Journal of Computer Applications

10. T. Razafindralambo, M. Erdelj, D. Zorbas, E. Natalizio Spread and shrink: Point of interest discovery and coverage with mobile wireless sensors. Journal of Parallel and Distributed Computing 2016 (11).

11. M. Rebai, M. Le berre, H. Snoussi, F. Hnaien and L. Khoukhi Sensor Deployment Optimization Methods to Achieve Both Coverage and Connectivity in Wireless Sensor Networks Computers and Operation Research, vol. 59, pp11-21, July 2015

12. M. Rebai, H. Murat Afsar, H. Snoussi Exact methods for sensor deployment problem with connectivity constraint in wireless sensor networks International Journal of Sensor Networks (IJSNET), Vol. 21, No. 3, 2016

13. L. Reynaud and I. Gurin Lassous Improving the performance of challenged networks with controlled mobility EAI International Conference on Ad Hoc Networks (Adhocnets), Sept. 2016, Ottawa, Canada.

14. A. Renzaglia, J. Dibangoye, V. Le Doze and O. Simonin, Multi-UAV Visual Coverage of Partially Known 3D Surfaces: Voronoi-based Initialization to Improve Local Optimizers Computing Research Repository, 2019

15. F. Tolba, C. Tolba and P. Lorenz Topology control by controlling mobility for coverage in wireless sensor networks. IEEE International Conference on Communications (ICC), May 2016, Kuala Lumpur, Malaysia.

16. Z. Wang, P. Chen, Z. Song, Y. Chen, K.L. Moore Formation control in mobile actuator/sensor networks SPIE, no. 435, Spie, 2005, pp. 706-717. 


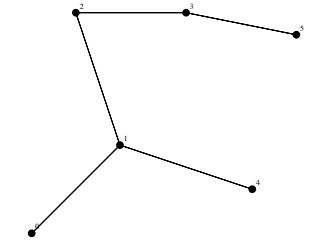

(a) GMs with $|K|=3$

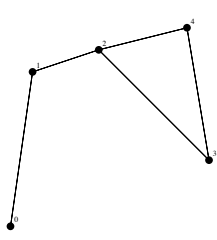

(b) GMs with $|K|=4$

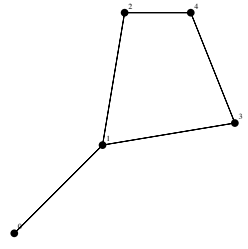

(c) GMs with $|K|=5$

Fig. 5: Topologies obtained with GMs for different numbers of sensor typologies.

17. Z. Yu, G. Shan, G. Xu and X. Duan Method of multi-sensor optimal deployment for area coverage. International Conference on Electronics Technology (ICET), Chengdu, China, 2018. 